\title{
BMJ Open Quality Atrial fibrillation detection using a automated electrocardiographic monitoring in a transient ischaemic attack service
}

\author{
Lucio D'Anna (D) , ${ }^{1,2}$ Oishi Sikdar, ${ }^{2}$ Suyin Lim, ${ }^{2}$ Dheeraj Kalladka, ${ }^{2}$ Soma Banerjee ${ }^{1,2}$
}

To cite: D'Anna L, Sikdar 0 , Lim S, et al. Atrial fibrillation detection using a automated electrocardiographic monitoring in a transient ischaemic attack service. BMJ Open Quality 2022;11:e001433. doi:10.1136/ bmjoq-2021-001433

- Additional supplemental material is published online only. To view, please visit the journal online (http://dx.doi.org/10. 1136/bmjoq-2021-001433).

Received 22 February 2021 Accepted 20 December 2021

Check for updates

(c) Author(s) (or their employer(s)) 2022. Re-use permitted under CC BY-NC. No commercial re-use. See rights and permissions. Published by BMJ.

${ }^{1}$ Department of Brain Sciences, Imperial College London, London, UK

${ }^{2}$ Department of Stroke and Neuroscience, Charing Cross Hospital, Imperial College London NHS Healthcare Trust, London, UK

Correspondence to

Dr Lucio D'Anna;

I.danna@imperial.ac.uk

\section{ABSTRACT}

Background The vast majority of the transient ischaemic attacks (TIA) services in UK reported significant delays in the initiation of the routine cardiac monitoring that may result in a significant number of missed atrial fibrillation (AF) paroxysms and increased long-term risk of recurrent stroke. Automated continuous ECG monitoring (ACEM) system has shown promising results in terms of $A F$ detection but it is unclear if ACEM improves AF detection in a rapid outpatient TIA service.

Objectives We assessed ACEM in patients with TIA with the aim to significantly reduce the delay to initiate the cardiac monitoring and to enhance the yield of AF detection in these patients. We also aimed to determine the impact of a more rapid initiation of ACEM on the 6-month risk of recurrent stroke/TIA.

Methods This is an observational, prospective before (phase 1: 1 July to 31 December 2018) versus after (phase 2: 1 January to 30 June 2019) study of the effect of ACEM, compared with routine initiation of $24 \mathrm{~h}-$ Holter ECG, in patients with TIA assessed in our service.

Results The phase $1(n=136)$ and phase $2(n=105)$ cohorts did not differ with regards to age, risk factors, duration of cardiac monitoring. The rate of newly detected AF was significantly higher in phase 2 compared with phase $1(9.52 \%$ vs $2.21 \%, \mathrm{p}<0.001)$. The 6 -month risk of recurrent stroke/TIA was significantly lower in phase 2 compared with phase 1 (7.4\% vs $1 \%, \mathrm{p}=0.018)$.

Conclusions Early initiation of ACEM improves AF detection after TIA in a rapid TIA service and is associated with a reduced risk of recurrent TIA/stroke.

\section{INTRODUCTION}

Transient ischaemic attacks (TIAs) are considered as part of the spectrum of ischaemic stroke given the common aetiological pathways and risk factors. ${ }^{1}$ Cardioembolism accounts for $17 \%-30 \%$ of all ischaemic stroke and $\mathrm{TIAs}^{2-4}$; in particular atrial fibrillation (AF) represents more than $50 \%$ of the causes of cardioembolism. ${ }^{5}$ It is noteworthy also that a similar proportion of patients with stroke might unknowingly have unrecognised $\mathrm{AF}^{6}$ Compared with patients without AF, those with the disorder have a five times higher risk of stroke. ${ }^{7}$ Hence, the rapid detection of AF after TIA is crucial because the use of oral anticoagulant can reduce the risk of stroke in patients with TIA with AF by up to twothirds. $^{89}$

Although in many healthcare systems there has been a mounting emphasis on developing dedicated services for patients with TIA, ${ }^{10-13}$ limited resources are allocated to perform cardiac evaluations rapidly in patients with TIA. The vast majority of the TIA services in the UK reported significant delays in the initiation of the routine cardiac monitoring that may result in a significant number of missed AF paroxysms. ${ }^{14}$

The automated continuous ECG monitoring (ACEM) system is a relatively new telemedicine service software based on $\mathrm{R}-\mathrm{R}$ interval dynamic analysis providing automated AF detection. ACEM has a 99\% sensitivity and specificity compared with the routine Holter ECG in the presence of manifest AF on ECG data. ${ }^{15} 16$ ACEM-Holter has also the advantage that can be performed at the stroke physician's office without sending the patient to the Cardiology Department.

The objective of this quality improvement report was to assess the effect of the use of ACEM in patients with TIA with the aim to significantly reduce the delay to initiate the cardiac monitoring and to enhance the yield of $\mathrm{AF}$ detection in these patients. We also aimed to determine the impact of a more rapid initiation of ACEM, compared with the standard of care such as the cardiac monitoring, on the 6-month risk of recurrent stroke/TIA in patients with TIA assessed in our rapid outpatient service.

\section{METHODS}

\section{Context}

Local general practitioners (GPs) in primarycare or accident and emergency departments can refer any patient they suspect had a TIA but whom they did not consider required 


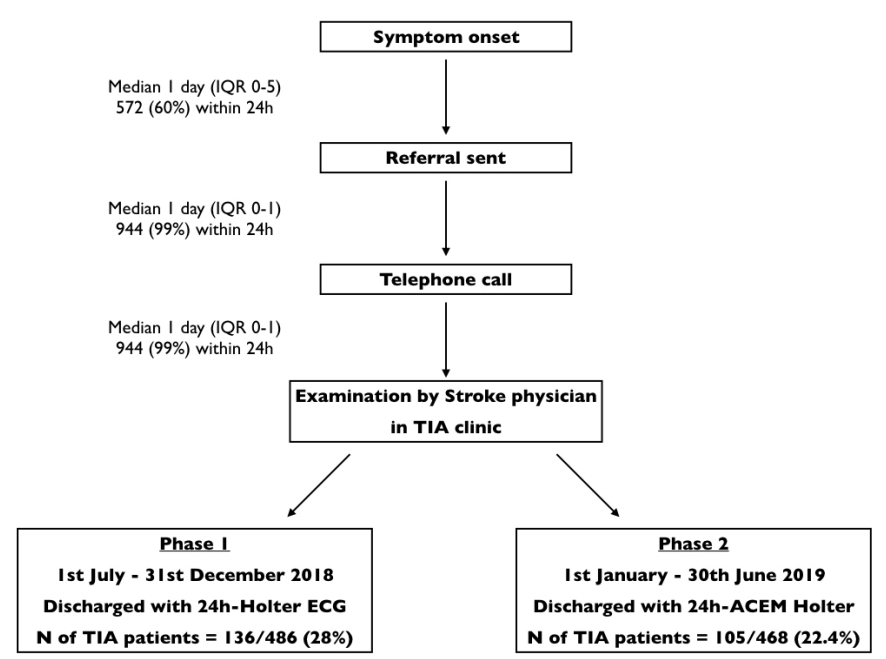

Figure 1 Time from symptom onset to telephone call and to examination in our TIA clinic. ACEM, automated continuous ECG monitoring; TIA, transient ischaemic attack.

immediate hospital admission, to the rapid TIA service at Charing Cross Hospital, Imperial College NHS Healthcare Trust, London (UK) (figure 1). These patients or the caregiver at home (usually by telephone) are then contacted by our team to arrange a clinic appointment within 24 hours of referral received. Our TIA clinic is organised to provide a standardised assessment to all our patients. On the same day, blood tests (lipid profile, red blood cell, white blood cell, platelet count, concentration of glucose, haemoglobin $\mathrm{A}_{1 \mathrm{c}}$, $\mathrm{C}$ reactive protein, creatinine), ECG, brain imaging (usually CT), and carotid ultrasound imaging and a clinical assessment by a stroke physician are obtained. Patients are discharged at home immediately after the assessment, unless the treating physician believes the patient requires urgent admission to our hyper acute stroke unit (HASU). Patients who are discharged with a diagnosis of TIA from our service are then contacted by our Cardiology Department, Imperial College NHS Healthcare Trust, to have a 24h-Holter ECG placed during a scheduled appointment.

\section{Local challenges in implementation}

The project had several implementation challenges with multiple stakeholder involvement for the large-scale use of ACEM in our TIA service. According to an internal audit conducted on 136 patients with TIA assessed in our TIA clinic between 1 July and 31 December 2018 (phase 1), the median time interval to initiate the 24h-Holter ECG was 62 days (IQR 13-111). The rate of newly detected AF was $2.21 \%(3 / 136)$ and $7.4 \%$ of the patients $(10 / 136)$ had a recurrent stroke/TIA within 6 months. We decided to use ACEM technology to significantly improve the delay to initiate the cardiac monitoring and to enhance the yield of $\mathrm{AF}$ detection in these patients and to reduce the 6-month risk of recurrent stroke/TIA.

Brainstorming meetings with all the TIA team members helped to develop a protocol and to find dedicated clinic space. Stroke clinical nurse specialists were trained to initiate the ACEM-Holter and to remove them after the recording. Stroke physicians were trained to use these new automated health tools, to send the traces via the secure internet connection and to read and interpret the results. We involved physicians and all the other health professionals to participate in this novel care delivery strategy process from very early on. This helped to create ideas to avoid the need for additional resources. Information Governance Department and the Information and Communication Technology Department at Imperial College NHS Healthcare Trust, London (UK) made a secure internet connection to send the ACEM-Holter traces to a central server (Apoplex Medical Technologies, Pirmasens, Germany) in order to respect privacy, security and data confidentiality of the patients involved in this project. They also ensured that all our infrastructure was in place to integrate this new technology.

\section{Study intervention}

Between 1 January and 30 June 2019 (phase 2) an ACEMHolter was applied for 24 hours to all the patients with TIA immediately after their assessment in our clinic at the stroke physician's office without sending the patient to the Cardiology Department. The day after patients returned in our clinic and the stroke physicians (LD or DK) sent the ACEM-Holter traces via a secure internet connection to a central server (Apoplex Medical Technologies, Pirmasens, Germany) where an automated AF episode detection algorithm was applied using the SRAclinic software. This software uses an algorithm that detects QRS complexes of the ECG data and then classifies them as being of atrial or ventricular origin and finally creates a list of R-R-intervals. ${ }^{15}$ To detect episodes of AF, the software performs a time series analysis of multiple mathematical parameters that are typical for an absolute arrhythmia during AF. The SRA algorithm consists of three main steps in identifying possible AF. In the end, this results in a final classification into one of the following three groups: (1) no evidence of AF; (2) increased risk of AF or (3) presence of manifest AF. SRA clinic detection of manifest AF episodes includes only episodes lasting $>30 \mathrm{~s}$. In the case of detected AF, the SRA service provides source of ECG rhythm strips which were reviewed by a trained stroke physician for confirmation of AF. SRA clinic has a $99 \%$ sensitivity and specificity compared with Holter ECG in the presence of manifest AF on ECG data. The final outcome of the traces analysis was sent from the central server to a secure email address and available to be read within few minutes. The clinical management as well as diagnostic procedures of all the patients with TIA, with and without AF, then followed routine practice of the treating physicians. Our team evaluates the risk of stroke and TIA at 6 months (in both phases) via the follow-up clinics during face-to-face interviews or through telephone calls. If the patient could not be contacted, a close relative or their family doctor is interviewed. 


\section{Patients}

The following inclusion criteria had to be fulfilled in both the two phases of the study: (1) age $\geq 18$ years old; (2) TIA diagnosis confirmed by a trained stroke physician; (3) no history of AF; (4) no AF on ECG performed in the TIA clinic; (5) no implanted pacemaker. Exclusion criteria were: (1) intracranial haemorrhage (2) TIA mimics ${ }^{17}$ (migraine aura, seizures, syncope, peripheral vestibular disturbance, transient global amnesia, functional/anxiety disorder, amyloid spells, subarachnoid haemorrhage, structural brain lesion and paroxysmal symptoms due to demyelination). We extracted event characteristics, patient demographics, risk factors and medical history from medical records. Data of consecutive patients were extracted using a prespecified case report file that encompassed patient characteristics including age, vascular risk factors, laboratory results, relevant medical history and medications. $\mathrm{CHA}_{2} \mathrm{DS}_{2}$-Vasc score was calculated for each patient.

\section{Definitions}

TIA was defined as a brief episode of neurological dysfunction caused by focal brain or retinal ischaemia, with clinical symptoms typically lasting less than 1 hour, and without evidence of acute infarction. ${ }^{18}$ By accepted convention, an episode lasting at least $30 \mathrm{~s}$ documented was considered as diagnostic for $\mathrm{AF}{ }^{19}$ High blood pressure was defined as systolic pressure $\geq 140 \mathrm{~mm} \mathrm{Hg}$ and/ or diastolic pressure $\geq 90 \mathrm{~mm} \mathrm{Hg}$, use of antihypertensive medication, and/or previous diagnosis of high blood pressure. Carotid stenosis was defined according to the Trial of ORG 10172 in Acute Stroke Treatment ${ }^{20}$ criteria as narrowing of the internal carotid artery lumen of $\geq 50 \%$ on carotid duplex ultrasound or angiography. Diabetes mellitus was defined as a history of diabetes that was confirmed in medical records, and/or use of insulin/oral hypoglycaemic agents, and/or random non-fasting blood glucose concentration $\geq 11.1 \mathrm{mmol} / \mathrm{L}$. Hypercholesterolaemia was defined as fasting total cholesterol serum level $\geq 5.18 \mathrm{mmol} / \mathrm{L} \quad(200 \mathrm{mg} / \mathrm{dL})$, and/or fasting low-density lipoprotein cholesterol serum level of $\geq 4.14 \mathrm{mmol} / \mathrm{L}(160 \mathrm{mg} / \mathrm{dL})$, and $/$ or use of lipid-lowering medications. Coronary heart disease was defined by a history of acute myocardial infarction, or angina pectoris, or coronary artery bypass graft, or percutaneous coronary intervention. The duration of the cardiac monitoring was automatically provided (hours) with the routine and ACEM-Holter analysis. The time to initiate the cardiac monitoring was calculated as the total time (days) from the point of the assessment in TIA clinic to the point of activation of the cardiac monitoring.

\section{Statistical analysis}

Data analysis was conducted using SPSS (V.26.0, IBM). Characteristics of the study population were described using medians and IQR or mean and SD or numbers with percentages. $\mathrm{P}<0.05$ was considered as significant. T-test or Mann-Whitney test were used to compare continuous

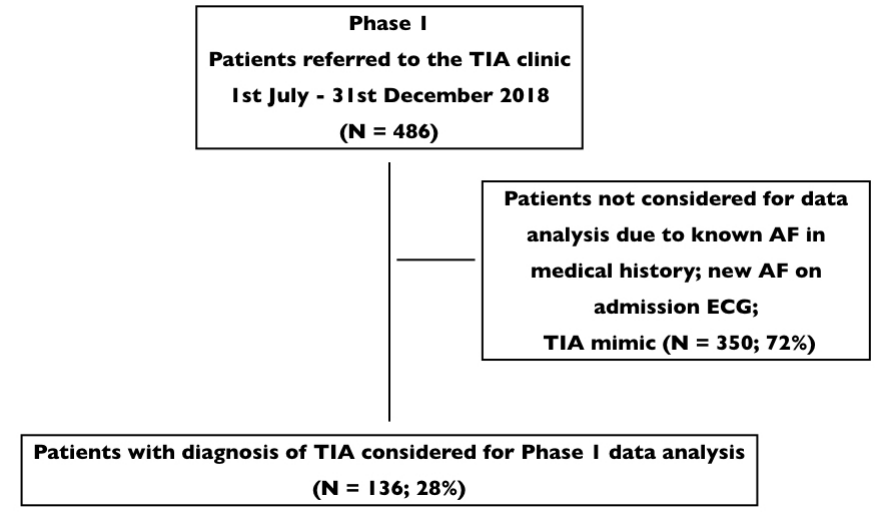

Figure 2 Patient recruitment in phase 1. AF, atrial fibrillation; TIA, transient ischaemic attack.

variables. For categorical variables, a $\chi^{2}$ or Fisher exact test was used to compare distributions.

\section{RESULTS}

Between 1 July and 31 December 2018 (phase 1), 486 patients were referred to our TIA clinic with suspected TIA and 136 (28\%) fulfilled the eligibility criteria (figure 2). During phase 2 (1 January to 30 June 2019) 468 patients with suspected TIA were referred to our service while 105 (22.4\%) fulfilled eligibility criteria (figure 3 ).

Baseline characteristics of the two cohorts of patients are reported in table 1 . Regarding the cardiac monitoring, two cohorts of patients did not show any statistically significant difference with regards to the duration of monitoring while the time interval to initiate the cardiac monitoring was significantly lower in phase 2 of the study compared with phase 1 (1 day (IQR 1-21) vs 62 days (IQR 13-111), $\mathrm{p}<0.001)$.

\section{Detection of AF in the two phases of the study}

Patients in phase 2 of the study had higher rate of newly detected AF than in the phase 1 cohort $(9.52 \%$ vs $2.21 \%$, $\mathrm{p}<0.001$ ) (table 2). Unadjusted HRs for the rate of newly detected AF in phase 2 versus in phase 1 was 4.67 (95\% CI 1.251 to $17.413, \mathrm{p}=0.022$ ).

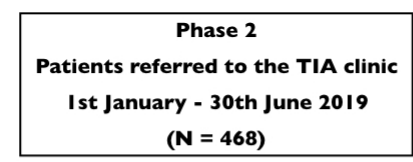

Patients not considered for data analysis due to known AF in medical history; new AF on admission ECG; TIA mimic ( $N=363 ; 77.6 \%)$

Patients with diagnosis of TIA considered for Phase 2 data analysis $(\mathrm{N}=105 ; 22.4 \%)$

Figure 3 Patient recruitment in phase 2. AF, atrial fibrillation; TIA, transient Ischaemic attack. 
Table 1 Baseline characteristics of the two cohorts of phase 1 and phase 2 patients

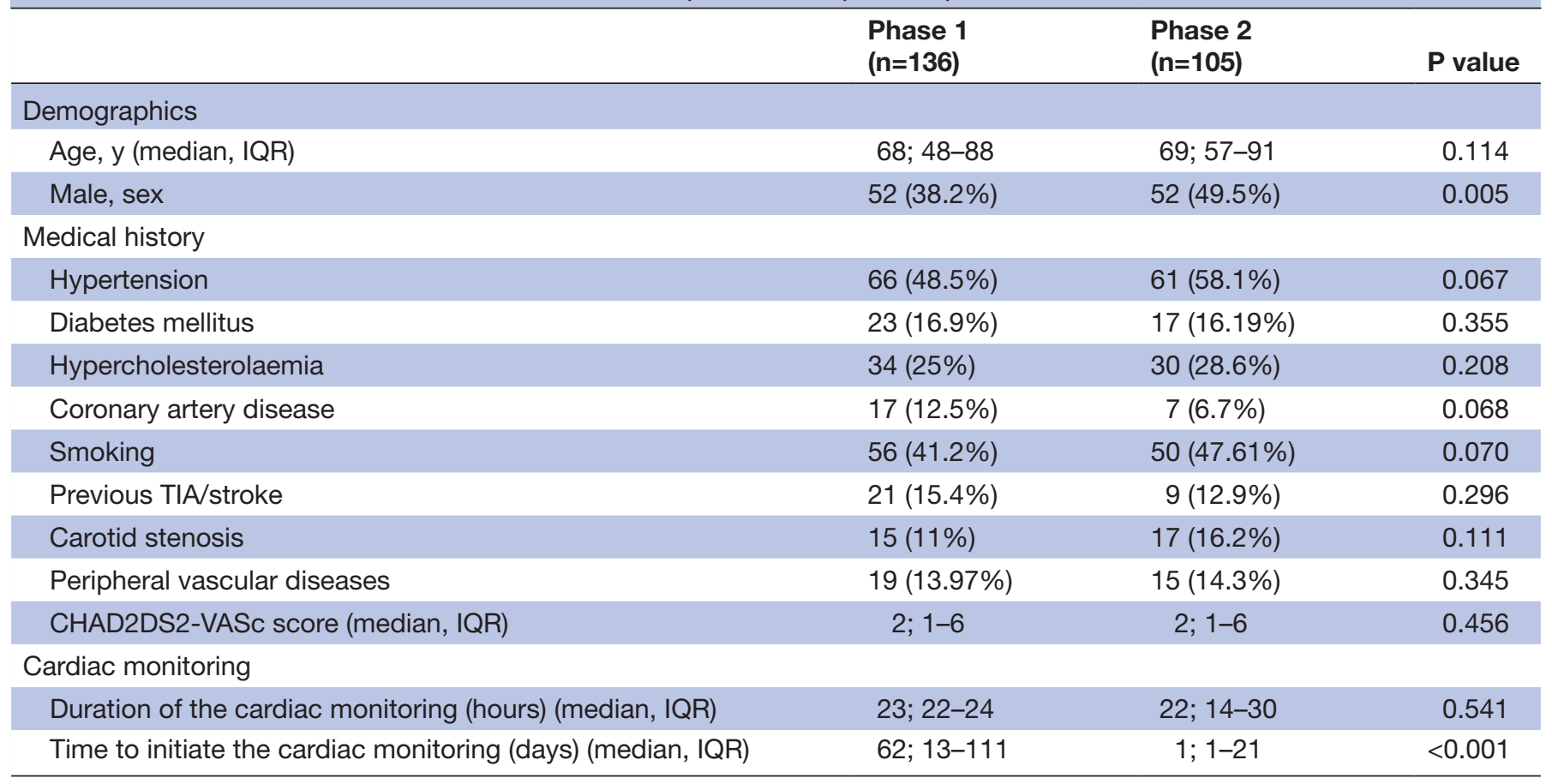

TIA, transient ischaemic attack.

\section{Six-month risk of stroke/TIA}

Patients in phase 1 showed a higher rate of stroke/TIA recurrence at 6 months $(\mathrm{p}=0.018)$ compared with the patients of phase 2 (online supplemental table 1). Unadjusted HRs for the rate of stroke/TIA recurrence at 6 months in phase 2 versus in phase 1 was 0.12 (95\% CI 0.015 to $0.962, \mathrm{p}=0.046$ ).

\section{DISCUSSION}

Our study highlights the importance of early initiation of cardiac monitoring for improving AF detection after a TIA in an outpatient TIA service. Our results are also consistent with the findings of the systematic review and meta-analysis by Sposato et al. ${ }^{6}$ The authors demonstrated that a mean time to the start of monitoring of 75 days was associated with a reduction in the detection rate of AF by over five times.

The second key finding of our study is that we observed that the 6-month risk of recurrent TIA/stroke fell significantly from phase $1(7.4 \%)$ to phase $2(1 \%)$. The earlier initiation of ACEM in phase 2 compared with the routine start of the Holter of phase 1 was associated with an almost

Table 2 Detection of atrial fibrillation in phase 1 and phase 2 of the study

\begin{tabular}{llll}
\hline & $\begin{array}{l}\text { Phase 1 } \\
(\mathbf{n}=136)\end{array}$ & $\begin{array}{l}\text { Phase 2 } \\
(\mathbf{n}=105)\end{array}$ & P value \\
\hline $\begin{array}{l}\text { Absolute no. of } \\
\text { detected cases }\end{array}$ & 3 & 10 & \\
\hline Absolute frequency & $2.21 \%$ & $9.52 \%$ & $<0.001$ \\
\hline
\end{tabular}

$90 \%$ reduction in the risk of 6 -month recurrent TIA/ stroke. It is also noteworthy that in phase 1 of the study, 4 out of $10(40 \%)$ of the recurrent stroke/TIA happened within 30 days after the first event. These findings are in line with previous studies. Lavallée $e t a l^{11}$ showed that a rapid assessment of patients with TIA reduced significantly the 90-day risk of stroke while Rothwell et $a l^{12}$ documented an $80 \%$ reduction in the risk of early recurrent stroke in patients with TIA who received a rapid outpatient assessment.

Overall, there are several technologies available for AF detection after a TIA or stroke that may vary significantly in their invasiveness and duration of monitoring. ${ }^{21}$ There are several core components of the ACEM-Holter successful implementation. One aspect is that compared with the routine Holter, the ACEM-Holter has the advantage that can be performed at the stroke physician's office without sending the patient to the Cardiology Department. This is the main reason why the time interval to initiate the cardiac monitoring was significantly lower in phase 2 compared with phase 1 of the study. Another very important advantage is also that this system was able to analyse automatically the ECG-traces sent and to provide the result within few minutes with a $99 \%$ sensitivity and specificity compared with Holter ECG in the presence of manifest AF on ECG data. ${ }^{15}$ This technology could be suited to be used in acute stroke patients that require bedside continuous electronically monitoring where the diagnosis of $\mathrm{AF}$ may require special attention and training by the stroke unit staff and can be often challenging or delayed. ACEM-Holter could be also adapted by local GPs in the primary-care where the screening for 
$\mathrm{AF}$ in the population is time-consuming and can often be difficult to access. Although our analysis did not include patients with recurrent TIAs as these patients are usually admitted in our HASU, we believe that also this subgroup of patients could take advantage from an early initiation of ACEM as previously documented. ${ }^{22}$

Our study has several limitations. First, the prospective observational study design may have introduced selection biases. Another important limitation is that the two groups of patients were enrolled in two different time periods. As reported previously, diagnostic criteria and investigations were identical in both study periods and detailed documentation of all relevant baseline clinical characteristics were obtained in both study periods. However, we cannot completely exclude that there were other confounders that affected the rates of AF detected and stroke/TIA recurrence rate.

\section{CONCLUSIONS}

In conclusion, the present study suggests early initiation of ACEM in a rapid outpatient TIA clinic improves AF detection and is associated with a reduced risk of recurrent TIA/stroke. Further studies are required to confirm the enhanced rate of AF detected using ACEM

Acknowledgements The authors would like to thank the following healthcare staff for their contributions: Tsering Dolkar and Siji Joy (stroke clinical nurse specialists), and Riann Kruger and Brooke Hall (data analysts for SSNAP).

Contributors LD served as quality improvement advisor and prepared initial draft of this manuscript, led the study and supervised implementation of changes. DK and SB played key role in physician's education and interventions. OS and SL data collection and analysis. LD assisted in statistical analysis. All authors contributed equally in the final approval of the manuscript. LD: guarantor.

Funding The authors have not declared a specific grant for this research from any funding agency in the public, commercial or not-for-profit sectors.

Competing interests None declared.

Patient consent for publication Not required.

Ethics approval This study involves human participants and was approved by the UK Health Research Authority. Informed consent of subjects was not needed as the data collected for the study were information collected as part of the routine care and only deidentified data were used in the research.

Provenance and peer review Not commissioned; externally peer reviewed.

Supplemental material This content has been supplied by the author(s). It has not been vetted by BMJ Publishing Group Limited (BMJ) and may not have been peer-reviewed. Any opinions or recommendations discussed are solely those of the author(s) and are not endorsed by BMJ. BMJ disclaims all liability and responsibility arising from any reliance placed on the content. Where the content includes any translated material, BMJ does not warrant the accuracy and reliability of the translations (including but not limited to local regulations, clinical guidelines, terminology, drug names and drug dosages), and is not responsible for any error and/or omissions arising from translation and adaptation or otherwise.

Open access This is an open access article distributed in accordance with the Creative Commons Attribution Non Commercial (CC BY-NC 4.0) license, which permits others to distribute, remix, adapt, build upon this work non-commercially, and license their derivative works on different terms, provided the original work is properly cited, appropriate credit is given, any changes made indicated, and the use is non-commercial. See: http://creativecommons.org/licenses/by-nc/4.0/.
ORCID iD

Lucio D'Anna http://orcid.org/0000-0002-6794-3850

\section{REFERENCES}

1 Easton JD, Saver JL, Albers GW, et al. Definition and evaluation of transient ischemic attack: a scientific statement for healthcare professionals from the American heart Association/American stroke association stroke Council; Council on cardiovascular surgery and anesthesia; Council on cardiovascular radiology and intervention; Council on cardiovascular nursing; and the interdisciplinary Council on peripheral vascular disease. the American Academy of Neurology affirms the value of this statement as an educational tool for neurologists. Stroke 2009;40:2276-93.

2 Murtagh B, Smalling RW. Cardioembolic stroke. Curr Atheroscler Rep 2006;8:310-6.

3 Khoo CW, Lip GYH. Clinical outcomes of acute stroke patients with atrial fibrillation. Expert Rev Cardiovasc Ther 2009;7:371-4.

4 Kishore A, Vail A, Majid A, et al. Detection of atrial fibrillation after ischemic stroke or transient ischemic attack: a systematic review and meta-analysis. Stroke 2014;45:520-6.

5 Arboix A, Alió J. Cardioembolic stroke: clinical features, specific cardiac disorders and prognosis. Curr Cardiol Rev 2010;6:150-61.

6 Sposato LA, Cipriano LE, Saposnik G, et al. Diagnosis of atrial fibrillation after stroke and transient ischaemic attack: a systematic review and meta-analysis. Lancet Neurol 2015;14:377-87.

7 Wolf PA, Abbott RD, Kannel WB. Atrial fibrillation as an independent risk factor for stroke: the Framingham study. Stroke 1991;22:983-8.

8 Secondary prevention in non-rheumatic atrial fibrillation after transient ischaemic attack or minor stroke. EAFT (European atrial fibrillation trial) Study Group. Lancet 1993;342:1255-62.

9 Ntaios G, Swaminathan B, Berkowitz SD, et al. Efficacy and safety of rivaroxaban versus aspirin in embolic stroke of undetermined source and carotid atherosclerosis. Stroke 2019;50:2477-85.

10 Giles MF, Rothwell PM. Transient ischaemic attack: clinical relevance, risk prediction and urgency of secondary prevention. Curr Opin Neurol 2009;22:46-53.

11 Lavallée PC, Meseguer $\mathrm{E}$, Abboud $\mathrm{H}$, et al. A transient ischaemic attack clinic with round-the-clock access (SOS-TIA): feasibility and effects. Lancet Neurol 2007;6:953-60.

12 Rothwell PM, Giles MF, Chandratheva A, et al. Effect of urgent treatment of transient ischaemic attack and minor stroke on early recurrent stroke (express study): a prospective population-based sequential comparison. Lancet 2007;370:1432-42.

13 Cancelli I, Janes F, Gigli GL, et al. Incidence of transient ischemic attack and early stroke risk: validation of the ABCD2 score in an Italian population-based study. Stroke 2011;42:2751-7.

14 Geraghty O, Korompoki E, Filippidis FT, et al. Cardiac diagnostic work-up for atrial fibrillation after transient ischaemic attacks in England and Wales: results from a cross-sectional survey. BMJ Open 2016;6:e012714.

15 Schaefer JR, Leussler D, Rosin L, et al. Improved detection of paroxysmal atrial fibrillation utilizing a software-assisted electrocardiogram approach. PLoS One 2014;9:e89328.

16 D'Anna L, Kar A, Brown Z, et al. Automated continuous electrocardiogram monitoring accelerates the detection of atrial fibrillation after ischemic stroke or transient ischemic attack on a hyper acute stroke unit. J Stroke Cerebrovasc Dis 2020;29:104669.

17 Nadarajan V, Perry RJ, Johnson J, et al. Transient ischaemic attacks: mimics and chameleons. Pract Neurol 2014;14:23-31.

18 Easton JD, Saver JL, Albers GW, et al. Definition and evaluation of transient ischemic attack. Stroke 2009;40:2276-93.

19 Kirchhof P, Benussi S, Kotecha D, et al. 2016 ESC guidelines for the management of atrial fibrillation developed in collaboration with EACTS. Eur J Cardiothorac Surg 2016;50:e1-88.

20 Adams HP, Bendixen BH, Kappelle LJ, et al. Classification of subtype of acute ischemic stroke. definitions for use in a multicenter clinical trial. TOAST. trial of ORG 10172 in acute stroke treatment. Stroke 1993;24:35-41.

21 Thijs V. Atrial fibrillation detection: fishing for an irregular heartbeat before and after stroke. Stroke 2017;48:2671-7.

22 Foschi M, Pavolucci L, Rondelli F, et al. Prospective observational cohort study of early recurrent TIA. Neurology 2020;95:e1733-44. 